\title{
Filigrane
}

Écoutes psychothérapiques

\section{Ignorer son chemin et accueillir le chaos : transmettre la psychanalyse et sa psychopathologie}

\section{Prado de Oliveira}

Volume 18, numéro 1, printemps 2009

Le corps. Sur le divan. Dans le fauteuil I

URI : https://id.erudit.org/iderudit/037719ar

DOI : https://doi.org/10.7202/037719ar

Aller au sommaire du numéro

Éditeur(s)

Revue Santé mentale au Québec

ISSN

1192-1412 (imprimé)

1911-4656 (numérique)

Découvrir la revue

Citer cet article

de Oliveira, P. (2009). Ignorer son chemin et accueillir le chaos : transmettre la psychanalyse et sa psychopathologie. Filigrane, 18(1), 40-47.

https://doi.org/10.7202/037719ar
Résumé de l'article

L'auteur utilise la problématique du corps dans un contexte particulier, soit pour présenter sa notion de la transmission psychanalytique. Pour lui, en tant que professeur, la transmission de la psychanalyse et de sa psychopathologie doit évoquer chez l'étudiant les tensions entre rêverie et rigueur, toujours basées sur l'expérience clinique la plus ardue et constante. La transmission de la psychanalyse, idéalement, devrait écarter toute langue de bois.

Malheureusement, l'existence de groupes et leur institutionnalisation exigent et imposent les jargons à travers lesquels les psychanalystes croient se reconnaître. Cette transmission évoquant les tensions entre rêverie et rigueur, l'auteur tente ici de l'illustrer en parlant de ce que comprend la psychanalyse du corps et de sa souffrance. 


\title{
Ignorer son chemin et accueillir le chaos: transmettre la psychanalyse et sa psychopathologie
}

\author{
prado de oliveira
}

\begin{abstract}
L'auteur utilise la problématique du corps dans un contexte particulier, soit pour présenter sa notion de la transmission psychanalytique. Pour lui, en tant que professeur, la transmission de la psychanalyse et de sa psychopathologie doit évoquer chez l'étudiant les tensions entre rêverie et rigueur, toujours basées sur l'expérience clinique la plus ardue et constante. La transmission de la psychanalyse, idéalement, devrait écarter toute langue de bois. Malheureusement, l'existence de groupes et leur institutionnalisation exigent et imposent les jargons à travers lesquels les psychanalystes croient se reconnaître. Cette transmission évoquant les tensions entre rêverie et rigueur, l'auteur tente ici de l'illustrer en parlant de ce que comprend la psychanalyse du corps et de sa souffrance.
\end{abstract}

$\mathrm{L}$ e psychanalyste travaille avec la libre association et, un autre mot pour le dire, l'attention flottante. Ce sont les propositions de Freud, qui a souvent exigé aussi à l'égard du travail analytique «la précision» ou «la froideur» du chirurgien. «Attention flottante» et «froideur chirurgicale» sont des postulats contradictoires. D'ailleurs, ce serait une question: est-ce que le chirurgien est animé exclusivement par cette précision et cette froideur?

Que ces postulats soient contradictoires, personnellement, cela ne me dérange pas. Nous avons un idéal de ce que nous devrions être ou faire et nous devons le concilier avec la réalité. Freud exprimait son idéal de l'analyse, mais il était loin de croire qu'idéal et réalité pouvaient se confondre.

En tant que professeur, je crois que la transmission de la psychanalyse et de sa psychopathologie doit évoquer chez l'étudiant ces tensions entre rêverie et rigueur, entre idéal et réalité, entre libre association et précision, toujours basées sur l'expérience clinique la plus ardue et constante. La tentation nosographique ne doit pas s'écarter de la clinique et du descriptif, sans lesquels la logique du délire devient délire de la logique. La transmission de la psychanalyse, idéalement, devrait écarter toute langue de bois. Malheureusement, l'existence de groupes et leur institutionnalisation exigent et imposent les jargons à travers lesquels les psychanalystes croient se reconnaître.

Cette transmission évoquant les tensions entre rêverie et rigueur, je tenterai de l'illustrer ici en parlant de ce que comprend la psychanalyse du corps et de sa souffrance, tout en ignorant à l'avance, tel Job, dont je traiterai plus loin, les détails du chemin que je prendrai pour le faire. 


\section{À propos du corps et de sa psyché. Souffrir, se plaindre}

Edipe ignorait son chemin. La tragédie de ce roi réside dans l'erreur du choix de son chemin et dans le fait de réaliser l'opposé de ce qu'il pense faire, autant que dans le meurtre de son père et le partage du lit de sa mère. Il croit choisir, alors que le destin le mène. Une version de son drame présente une Jocaste qui exige la clémence à l'égard du couple qu'elle forme avec son fils. Elle lance à Tirésias :

«En plus des douleurs, ne crée pas des durs soucis

«Et ne m'annonce pas

«De lourdes attentes pour l'avenir,

«Car les dieux immortels n'ont pas fixé

«Que toujours également la discorde s'avancerait sur la terre

«Attachée aux mortels,

«Ni l'amour non plus. Mais, jour après jour,

«Les dieux fixent (ou l'un ou l'autre)

«Et tes prédictions, Seigneur, (Zeus peut)

«Ne pas les réaliser toutes.» (Bollack, Judet de la Combe,

Wismann, 1977)

Ainsi, Jocaste sollicite la clémence, l'exige même.

À l'instar de Jocaste, mais de façon différente, Clémence clame sa douleur. Elle m'a été référée par des collègues médecins d'un important centre hospitalier parisien en raison de sa «fibromyalgie», terme impressionnant à l'époque, qui n'est pas attesté dans le Trésor de la langue française informatisé (http://atilf.atilf. fr/tlf.htm). Il signifie «douleur généralisée dans le corps ». Clémence se plaint de douleurs à la plante des pieds, à chaque articulation de son misérable corps haut perché sur des talons aiguilles, de sa pauvre main d'institutrice dans les collèges privés, où elle corrige des centaines de copies par jour, en portant des annotations à chacune. Elle se plaint de douleurs à la mâchoire, aux yeux, aux oreilles, à la tête, aux racines des cheveux si bien coiffés en boucles, lui donnant un air adolescente malgré ses cinquante ans. Des douleurs encore aux genoux, aux jambes, sur le bas ventre jamais touché par une main d'homme autre que celle de son médecin. Bien entendu aussi, des douleurs lombaires à un dos sur lequel elle ne peut jamais compter ni se reposer, sauf en présence de son acupuncteur, qu'elle voit régulièrement, deux à trois fois par semaine.

— «Pensez-vous venir aussi souvent à ses séances de psychothérapie?»

— «Malheureusement, non.»

— «C'est dommage. Depuis combien de temps votre «fibromyalgie» a-t-elle été détectée par les médecins?» 
- «Récemment. Un jour où mon acupuncteur n'avait pu me piquer que de l'une de ses mains et que je m'en étais plainte, il m'a conseillée d'aller voir un médecin. Indignée, je le fis immédiatement. »

- «Et cet acupuncteur lui-même, depuis combien de temps le voyez-vous?»

- «Des années.»

Seule au monde, elle n'a que lui, ses élèves et sa hiérarchie.

- «Absolument seule?»

- «Oui, complètement. »

- «Pas de parents, pas de fratrie?»

- «Si, si, mais je suis fâchée avec eux depuis longtemps; je ne les fréquente plus, sauf très rarement une sœur de ma mère.»

- «Votre tante?»

- «Ah... oui, c'est ça.»

- «Mal au père, mal à la mère, mal aux frères et sœurs?

- «Vous avez une drôle de façon de parler!»

- «C'est curieux, vous n'avez mal qu'à l'extérieur!»

- «Ah, je ne vous ai pas parlé du reste! Le médecin m’a dit de vous en parler. J'ai aussi mal au ventre et très mal aux poumons.»

- «Vous fumez?»

- «C'est ça le problème. Jusqu'à quatre paquets par jour.»

- «Quatre?!»

- «Quand mon ami n'est pas là.»

- «Ah, vous avez un ami? Je ne l'ai pas compris!»

- «C'est qu'il n'est jamais là !»

- «Mais, parfois!»

- «Oui, mais il boit tout le temps!»

- «Et vous buvez avec lui ?»

- «Oui, et je fume plus aussi. Il fume des cigares et j’aime bien ça.»

- «Est-ce que vous avez une idée des raisons de votre médecin de vous envoyer vers moi?»

- «Aucune».

Clémence souffre mille morts, elle souffre mort, amour et passion, d'un seul trait. Ou, plutôt, de mille traits éparpillés dans toutes les fibres de son corps. Qu'est-il arrivé de l'élaboration de son complexe familial?

Dans mon expérience clinique, la difficulté de nommer directement un lien de parenté et de prendre un détour pour le faire correspond au démantèlement de la 
famille. Ici, Clémence ne garde pas une relation lointaine avec l'une de ses tantes, mais avec «une sœur de sa mère». Certes, elle peut reconnaître ce que je lui dis, après un court délai, mais ce n'est pas spontané. Dans d'autres cas, cette reconnaissance est impossible.

Démantelée, la famille est incapable de jouer son rôle de pare-excitation, élargi au-delà de ce que la mère aurait eu à faire. Le sujet est ainsi pris entre excitations internes et externes, sans médiation. Il s'ensuit un corps souffrant et un appareil psychique écartelé entre imaginaire et identifications spéculaires. Sa vie devient une longue expiation.

J'en veux au médecin qui m'a envoyé Clémence. En fantasme, je suis prêt à l'égorger, je demanderai à mon médecin-chef de s'en occuper, je veillerai à ce qu'il soit rayé de l'ordre des médecins. Je connais déjà son argument. Il est débordé de patients, il ne peut pas s'occuper de tout le monde. Moi non plus, je ne peux m'occuper de tout le monde. À mon tour, je me mets à me plaindre. Pire, je suis prêt à dénoncer, à accuser, à tuer, pour me venger.

Il y a des psychanalystes qui n'aiment pas que l'on expose des cas cliniques et que l'on parle de soi. Pourtant, c'est la base même de l'enseignement de Freud. Des cas, il en expose depuis le début et toujours. De soi-même, pouvons-nous ne pas en parler, tout en rêvant d'objectivité, précision et froideur? Freud parle de soi à ses patients. Il leur parle même de ses autres patients.

\section{Plainte et psychanalyse}

De quoi le corps des patients tels que Clémence se plaint-il ?

Dans de rares occasions, Freud traite de la plainte en tant que telle. «La femme qui déplore bien haut pour son mari qu'il soit lié à une femme si peu à la hauteur, veut, à vrai dire, accuser le mari de n'être pas à la hauteur, en quelque sens qu'on l'entende.... Le comportement des malades, lui aussi, devient maintenant de beaucoup plus compréhensible. Leurs plaintes sont des plaintes accusatrices, conformément au vieux sens du mot allemand — ihre Klagen sind Anklagen — ils n'ont pas honte et ne se cachent pas, car tout ce qu'ils disent d'abaissant sur euxmêmes est, au fond, dit d'un autre; et ils sont bien loin de témoigner, à l'égard de leur entourage, l'humilité et la soumission qui seules conviendraient à des personnes si indignes; bien au contraire, ils sont tourmenteurs au plus haut point, toujours comme s'ils étaient atteints et comme si un grand tort leur avait été fait. » (Freud, 1915, 267)

En vieux français, le mot de plainte correspond à «expression d'un mécontentement». «Se plaindre» est alors synonyme «d'accuser», «faire plainte», écrit Chrétien de Troyes. Et il en est toujours ainsi, encore de nos jours. (Voir le Trésor de la langue française informatisé, déjà mentionné, mais aussi Le Grand Robert de la langue française, informatisé, se plaindre: déposer une plainte.)

Dans ses réflexions sur le travail de la mélancolie, Freud souligne «que les plaintes identiquement formulées, fatigantes par leur monotonie, proviennent 
cependant chaque fois d'un autre fondement inconscient. ( (Freud, 1915 [1917], 267 et 275). Je souligne le mot travail. Je souligne aussi cette dernière phrase. Car, pour Freud, la mélancolie en tant que telle importe moins que le travail de la mélancolie, de même que le rêve importe moins que le travail du rêve. Et le fait de provenir à chaque fois d'un autre fondement inconscient indique le caractère surdéterminé de ce travail. Je souligne aussi ce dernier mot de surdétermination, car ce concept introduit un véritable séisme dans la pensée et pour la compréhension des phénomènes de l'âme.

Le terme «inconscient» est largement utilisé à l'époque de Freud. Celui de sexualité aussi. L'intérêt de l'étude des rêves apparaît déjà avec Aristote, sinon avant. Mais le concept de surdétermination, pourtant au fond si populaire, était exclu du monde de la pensée. Son apparition date de l'avènement de la psychanalyse. Freud l'introduit en psychopathologie. Celui qui se plaint, accuse. Bien entendu, celui qui accuse se plaint aussi. Plainte et accusation vont de pair, surtout la plainte répétitive, immuable, fatigante, qui paralyse celui qui l'écoute, plutôt que de lui permettre d'intervenir pour soulager la douleur qu'elle recouvre.

Il suffit de lire les lettres de Freud. Il se plaint: de Stekel, de Jung, de Ferenczi, d'Adler, de Rank, du manque supposé de reconnaissance de son époque envers son œuvre, des persécuteurs qui le menacent à chaque conférence, à chaque critique de ses textes, des «attaques contre la psychanalyse». Les lettres de Freud à ses principaux correspondants, Jung et Ferenczi, encore une fois, mais aussi Abraham, Pfister, Jones et d'autres en témoignent. Et même pire: Freud se plaint de Jung à Ferenczi en même temps qu'il se plaint de Ferenczi à Jung. Il se plaint de Jones à Ferenczi au même moment qu'il se plaint de Ferenczi à Jones. Freud est peut-être stoïque devant son cancer de la mâchoire, mais il ne l'est pas devant les avatars de la discipline qu'il inaugure.

\section{D'autres plaintes}

Mélancolie et paranoïa sont intimement liées. Le caractère obséquieux du mélancolique ou du paranoïaque cache mal la mégalomanie et le sentiment de persécution qui leur sont sous-jacents. Melanie Klein les relie ainsi : la phase de sadisme maximal est celle des sentiments de persécution les plus intenses (Klein, 1930, 277 ; Klein, 1932, 179; Klein, 1935, 318). Ce qu'elle écrit est très expressif : Höchstblüte des Sadismus, apogée du sadisme, la plus haute prospérité du sadisme, ou, encore, littéralement, « la plus haute fleur du sadisme». Il y aurait une fleur du sadisme. Et, en effet, le véritable sadisme est feutré et délicat, plus que violent. Les femmes bottées et munis de fouets relèvent de l'imaginaire russoaméricain, bien plus que de la Vienne fin de siècle, ou de l'Angleterre d'avantguerre.

Ceux qui ont travaillé en santé mentale apprennent à résister à cette douleur infligée par les patients mélancoliques, irréductibles dans leurs plaintes. Ils nous offrent les fleurs de leurs souffrances comme des fleurs du mal, mais fleurs quand même. 
J'évoque ici un cas peu connu de Lacan, celui de Paule Mulatier qui l'a fréquenté en cure analytique pendant quatre ans. Elle aurait pu souffrir de «fibromyalgie». À la fin de cette période, vers 1950, un internement psychiatrique est décidé. Paule commence son récit avec les mots de Daniel Paul Schreber, à peu de choses près : «La cure de sommeil commença dans des mauvaises conditions. » (Schreber, 1901, 48)

Paule était prisonnière, elle allait mourir de pourriture, signe et châtiment de son infamie, on ne la laisserait sortir que quand sa puanteur serait devenue insupportable, elle était une créature sordide, elle irait aussitôt en enfer. «Les journaux annonceraient en première page : "La soi-disant Sœur Marie de la Trinité, Paule de Mulatier, a été trouvée morte, pourrie, dans une chambre de l'hôpital psychiatrique de Bonneval.' Ils diraient que j'étais une fausse religieuse et détailleraient toutes les faussetés de ma vie. Et ils auraient raison.... Le scandale allait rejaillir sur l'Église, le Pape et les évêques, l'état religieux en général, ma Congrégation, mon couvent, ma famille. Et tout le monde le saurait.... Je voyais déjà les énormes caractères de tous les journaux - et la photo qui me montrerait pourrie dans un coin de la chambre: "L'ex Sœur Marie de la Trinité".» (Mulatier, 2003, 19 et 3235) Comme Schreber entend des voix qui disent de lui, d'un ton moqueur : "voilàt-il pas un ex-président de chambre...» (Schreber, 1901, 151)

Paule Mulatier, obscène, est au centre du monde, comme Schreber. Ses souffrances détruiraient tout son entourage. Elles expriment son agressivité généralisée.

\section{«Comment?» Comment les souffrances expriment-elles l'agressivité?}

La psychiatre qui rencontre Paule une demi-douzaine de fois a du mal à identifier un diagnostic convenable. Troubles obsessionnels graves, pense-t-elle. Comment savoir? Ces troubles sont si présents dans la mélancolie et dans la paranoïa, autant que les sentiments d'indignité de soi et de persécution dans les névroses obsessionnelles et dans les hystéries. Freud commence ses exposés des Études sur l'hystérie en écrivant sur des patientes hystériques qui évoluent rapidement vers la psychose, Schreber évolue de la démence paranoïde à la paranoïa et de la paranoïa à une sorte de transvestisme obsessionnel. La nosographie et le pronostic ont la même utilité pour la psychanalyse que l'astrologie pour l'astronomie.

Dans le cas de Paule, s'agit-il d'une guérison induite par la psychiatrisation ou par la cure analytique préalable, s'interroge sa psychiatre. Comment savoir? Il n'est pas exclu que l'une n'aurait pas pu aller sans l'autre. Mais cette psychiatre écrit sur «l'épreuve de Job» de Marie de la Trinité. La psychiatre la croit guérie puisque Paule est devenue psychothérapeute. Je préfère revenir à Kraepelin ou Freud: la mélancolie paranoïde de Paule se serait résolue grâce à ses nouvelles fonctions dans son couvent et à sa nouvelle formation comme psychothérapeute. Dans ce couvent, elle trouve une mère supérieure et un père confesseur, c'està-dire une configuration où elle peut équilibrer son élaboration œdipienne défaillante et la relancer, au moins dans un premier temps, dans un cadre précis. 


\section{Job}

La psychiatre de Paule Mulatier évoque «l'épreuve de Job» pour décrire les souffrances de sa patiente. C'est vite dit, car il y a un abîme entre le cadre des tragédies grecques et les entretiens singuliers des religions monothéistes. Edipe ne se plaint pas ni n'accuse. Les constellations des tragédies impliquent toujours plusieurs médiateurs et intermédiaires, ce qui n'est pas le cas dans les dialogues monothéistes entre un sujet et son dieu.

Le livre de Job vient après celui de Jérémie, dont le titre aurait dû être «Comment?». Comment osez-vous, ô Dieu? Un «comment» d'indignation, accusateur, qui n'est pas une vraie question. La religion juive inscrit la plainte et l'accusation au centre de sa démarche. Ni Edipe, ni Oreste, ni Prométhée, ni Héraclès ne se plaignent, malgré leurs souffrances. Ils ne se plaignent en tout cas pas de leurs dieux, ni à eux, ils n'entament pas non plus un débat ou une conversation avec eux. Ils prient et ils se courbent.

Jérémie, lui, accuse avec violence, il terrifie, il se plaint. De son peuple, de son dieu, de tout et rien. Jérémie fait feu de tout bois. Lisez Jérémie : il ne craint pas l'incohérence, la répétition, la monotonie. C'est «le livre des lamentations», autre nom pour la plainte. Jérémie crée un genre littéraire nouveau. Là où Edipe se repentit, Jérémie accuse et se plaint.

Job vient dans son sillage, mais énormément de choses ont changé. D'abord, Job n'est pas un Juif. «Il y a un homme au pays d'Outs », c'est la première phrase du Livre de Job. Il vient de quelque part au sud de la mer Morte, d'une nation ennemie d'Israël et de Juda. «Il y a un homme au pays d'Outs. Il s'appelle Job. C'est un homme bien, un homme droit. Il craint Dieu. Il évite le mal.»

Et Dieu se plaint à Job de sa propre divinité, en l'accusant de son humanité à lui. «Qui es-tu pour noircir mes desseins/de tes mots d'ignorant ?/ceins tes reins comme un homme/je vais t'interroger/instruis-moi :/où étais-tu quand j'ai fondé la terre?» (Job, 38-2 à 42.8).

C'est peut-être la première fois dans l'histoire de la littérature où Dieu se plaint et accuse et demande à être instruit, où un père apprend de son fils. Dieu reconnaît le bien-fondé des arguments de Job. Il faudra Paul et son christianisme, pour exiger le repentir comme résolution de la plainte et de l'accusation. Il faudra Paul pour que se fasse l'intégration entre la tragédie grecque et la légende judéo-chrétienne. Même si l'une et l'autre sont toujours prêtes à se séparer et se désintégrer face à la douleur et à l'acceptation de l'inévitable. Job est clair: "l'homme ignore son chemin et accueille le chaos.» (Job, 3-23)

Ce que ne savaient ni Clémence, ni Paule Mulatier, ni même sa psychiatre, toujours prêtes à imposer du sens là où il y en a pas, où il n'a jamais existé et jamais n'existerait peut-être. Le sens ne peut être que véritable interrogation.

\section{Un mot de fin}

J'essaierai de résumer maintenant mon point de vue sur la transmission de la psychanalyse et de la psychopathologie. Je pars d'un cas de mon expérience 
clinique, parfois, comme ici, j'essaye de le relier à un cas de l'expérience clinique d'un collègue. J'essaye de relier mes cas cliniques à une formation culturelle plus générale. Mon but est que l'apprentissage de la psychopathologie soit un exercice vivant de réflexion sur l'expérience de ceux que j'ai à former, à l'université ou en supervision. Cette réflexion doit s'inscrire dans une pensée plus large sur la culture et amener à des questionnements.

Dans cette perspective, les étudiants ou jeunes analystes en supervision sont des Sphinges qui attendent un maître ignorant son chemin autant que le leur. La Sphinge ne sait pas que des chemins existent. Elle attend. Edipe aussi ignore son chemin et abrite le chaos. De l'abriter, c'est la condition pour enfin trouver sa route vers Athènes, ville de justice et pardon.

Écrasée par un surmoi archaïque, abritant une sorte de chaos où imaginaire et réel se rencontraient dans son corps, Clémence abandonne un traitement qu'elle n'a jamais demandé et encore moins souhaité, faute de pouvoir le penser. Paule Mulatier trouve une sorte d'inscription symbolique dans son couvent. D'avoir vaincu leur dieu, Jérémie et Job ont guéri de leurs plaintes. Les étudiants ou analystes en supervision continuent à poser des questions et les maîtres sont condamnés à essayer d'y répondre, faute de leur apprendre à les répondre euxmêmes et d'eux-mêmes. Cette situation constitue un complexe familial irrésolu et, dans l'état actuel de choses, insoluble. Le drame d'Edipe, en vérité, n'a pas de fin.

prado de oliveira

107 , rue mouffetard

75005 Paris

pradodeoliveira@free.fr

\section{Bibliographie}

La Bible, Desclée de Brouwer, Paris, 1985, traduction A. Chouraqui.

Bollack, J., Judet de la Combe, P., Wismann H., 1977, La réplique de Jocaste: sur les fragments d'un poème lyrique découvert à Lille (Papyrus Lille 76a, b et c), Cahiers de Philologie, Centre de Recherche Philologique de l'Université de Lille III, Publications de l'Université de Lille III, Éditions de la Maison des Sciences de l'Homme, Lille.

Freud, S., 1915 [1917], Deuil et mélancolie, in CEuvres complètes, XIII, PUF, Paris, 1988, trad. J. Altounian, A. Bourguignon, P. Cotet, A. Rauzy.

Klein, M., 1930, L'importance de la formation du symbole dans le développement du moi, in Essais de psychanalyse, 1921-1945, Payot, Paris, 1968, trad. M. Derrida, 263-282.

Klein, M., 1932, Les rapports entre la névrose obsessionnelle et les premiers stades de la formation du surmoi, in $L a$ psychanalyse des enfants, PUF, Paris, 1969, trad. J. B. Boulanger, 163-190.

Klein, M., 1935, Contribution à l'étude de la psychogénèse des états maniaco-dépressifs, in Essais de psychanalyse, 1921-1945, Payot, Paris, 1968, trad. M. Derrida, 311-340.

Mulatier, P. de, 2003, Marie de la Trinité : de l'angoisse à la paix. Relation écrite pour Jacques Lacan, présentation par le $\mathrm{D}^{\mathrm{r}}$ Jacqueline Renaud, Arfuyen.

Schreber, D. P., 1901, Mémoires d'un névropathe, Seuil, Paris, 1975, traduction P. Duquenne et N. Sels.

Job, 2001, Gallimard, Paris traduction de P. Alferi et J.-P. Prévost. 